\title{
Development of a tomato harvesting robot used in greenhouse
}

\author{
Wang Lilili, ${ }^{1,}$ Zhao Bo ${ }^{2}$, Fan J inwei ${ }^{1 *}$, Hu Xiaoan², Wei Shu², Li Yashuo², \\ Zhou Qiangbing ${ }^{2}$, Wei Chongfeng ${ }^{2}$
}

(1. College of Mechanical Engineering and Applied Electronics Technology, Beijing University of Technology, Beijing 100124, China; 2. State Key Laboratory of Soil-Plant-Machine System Technology, Chinese Academy of Agricultural Mechanization Sciences,

Beijing 100083, China)

\begin{abstract}
A tomato harvesting robot was developed in this study, which consisted of a four-wheel independent steering system, a 5-DOF harvesting system, a navigation system, and a binocular stereo vision system. The four-wheel independent steering system was capable of providing a low-speed steering control of the robot based on Ackerman steering geometry. The proportional-integral-derivative (PID) algorithm was used in the laser navigation control system. The Otsu algorithm and the elliptic template method were used for the automatic recognition of ripe tomatoes, and obstacle avoidance strategies were proposed based on the $\mathrm{C}$-space method. The maximum average absolute error between the set angle and the actual angle was about $0.14^{\circ}$, and the maximum standard deviation was about $0.04^{\circ}$. The laser navigation system was able to rapidly and accurately track the path, with the deviation being less than $8 \mathrm{~cm}$. The load bearing capacity of the mechanical arm was about $1.5 \mathrm{~kg}$. The success rate of the binocular vision system in the recognition of ripe tomatoes was $99.3 \%$. When the distance was less than $600 \mathrm{~mm}$, the positioning error was less than $10 \mathrm{~mm}$. The time needed for recognition of ripe tomatoes and pitching was about $15 \mathrm{~s}$ per tomato, with a success rate of about $86 \%$. This study provides some insights into the development and application of tomato harvesting robot used in the greenhouse.
\end{abstract}

Keywords: tomato harvesting robot, four-wheel independent steering, automatic navigation, binocular stereo vision system, obstacle avoidance, greenhouse

DOI: $10.25165 /$ j.jiabe.20171004.3204

Citation: Wang L L, Zhao B, Fan J W, Hu X A, Wei S, Li Y S, et al. Development of a tomato harvesting robot used in greenhouse. Int J Agric \& Biol Eng, 2017; 10(4): 140-149.

\section{Introduction}

Tomato is one of the most popular and widely grown

Received date: 2017-01-13 Accepted date: 2017-04-18

Biographies: Wang Lili, $\mathrm{PhD}$ candidate, Engineer, research interests: agricultural machinery automatic detection and control, robot technology, Email: lily979608886@163.com; Zhao Bo, Research Fellow, research interests: intelligent agricultural mechanization, Email: zhaoboshi@126.com; Hu Xiaoan, Research Fellow, research interests: agricultural mechanization, Email: huxiao_an@sina.com; Wei Shu, Engineer, research interests: image processing, Email: 79881708@qq.com; Li Yashuo, Engineer, research interests: image processing, Email: 449183787@qq.com; Zhou Qiangbing, research interests: agricultural mechanization, Email: 309411698@qq.com; Wei Chongfeng, research interests: agricultural mechanization, Email: 20287586@qq.com.

*Corresponding author: Fan Jinwei, Professor, research interests: precision processing and servo control. Beijing University of Technology, Beijing 100124, China. Tel: +86-10-64882652, Email: jwfan@bjut.edu.cn. vegetables in the world, with an annual production of about 60 million tons ${ }^{[1-3]}$. Manual harvesting of tomatoes is laborious, time consuming and inefficient, making it somewhat impractical for large-scale plantations. Clearly, this problem becomes more remarkable with the increase of labor cost in China. Thus, automatic harvesting has emerged as a promising alternative to manual harvesting, and much effort has been devoted to the development of tomato harvesting robot. However, as tomato is very soft and prone to bruising, many technical challenges remain to be solved before the practical application of the tomato harvesting robot.

A wide variety of robots have been developed for harvesting tomato, cucumber, grape and orange in Japan, Holland, England, France, Italy, the USA, Israel, etc. ${ }^{[4,5]}$. Kyoto University developed a tomato harvesting robot 
with a 5-degree of freedom (DOF) manipulator. Okayama University developed a 7-DOF robot consisting of a moving system, a vision system, an end effector, a manipulator and a control system. Later, a new tomato harvesting robot consisting of a vision system, a manipulator, a control system and a rotating arm was developed. The time needed from recognition to pitching was about $15 \mathrm{~s}$ per tomato, with a success rate of $50 \%-70 \%{ }^{[6]}$. Moreover, the Institute of Agricultural Mechanization Science of Korean also developed a series of tomato harvesting robots. The surface color of tomato could be precisely determined by the vision system, which thus enabled the robot to selectively pick ripe tomatoes. However, a potential drawback of these robots is their slow reaction and clumsy movement ${ }^{[7]}$. In China, much progress has been made in the research of tomato harvesting robot, such as the manipulator, image recognition, and motion control ${ }^{[8-11]}$. In general, current robots are not intelligent enough, and the success rate and pitching rate fall far short of what is expected. In addition, most tomato harvesting robots are designed for harvesting tomatoes at a certain height along the guide rail. The two-wheel drive control and differential steering control cannot meet the demand for the automatic harvesting of fruits and vegetables. In addition, the picking arms are generally replaced by industrial robotic arms, which suffer from the drawback of high cost and complexity of control. It takes 3-7 s to pick a citrus, $15 \mathrm{~s}$ to pick a melon, $10 \mathrm{~s}$ to pick a cucumber, and 1 min to pick an eggplant, respectively, and the harvest rate is less than $90 \%$. The fruits and vegetables are identified mainly by color characteristic, the grey degree threshold and the geometrical shape. These algorithms are likely to be affected by light and environmental factors, and it is difficult to identify overlapping fruits.

To address these problems, a tomato harvesting robot used in the greenhouse was developed in this study, which was capable of automatic navigation, recognition and positioning of ripe tomatoes, avoiding obstacles, and harvesting. The image recognition algorithm, the chassis walking control method and the picking control method are discussed in this paper.

\section{System compositions and workflow}

In view of the small spacing, planting density, and the complex working environment, the overall objective is to design a light, small and flexible automatic harvesting robot. The robot developed in this study can be used to harvest ripe tomatoes in the greenhouse, which consists mainly of a four-wheel independent steering chassis, a 5-DOF harvesting system, a navigation system, and a binocular vision system, as shown in Figure 1. It is a multipurpose harvesting robot, which has the potential to be used for the harvesting of various kinds of fruits after a simple modification of its end effector and the control program.

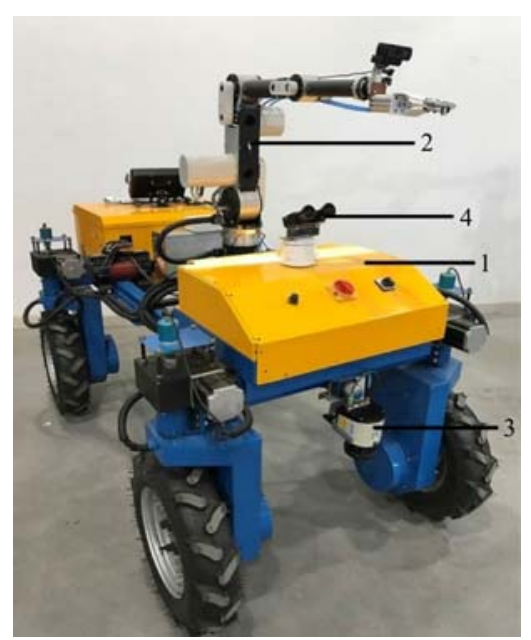

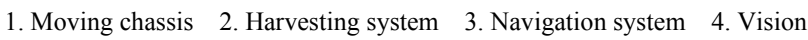
system

Figure 1 Overall structure of the tomato harvesting robot

The workflow of the robot is as follows: the robot moves automatically along the path predetermined by the navigation system, and the binocular stereo vision system installed on the mechanical arm begins to detect whether there are ripe tomatoes in the operation area based on the color difference between the ripe tomatoes and the background. Once ripe tomatoes are detected, the robot stops moving, and then the spatial location of ripe tomatoes and obstacles that may impede the harvesting are further determined by the vision system, based on which the mechanical arm can guide the end effector to reach the target position by using different strategies. The tomato is clamped and detached from the plant, and then placed into the bucket. After that, the mechanical arm is reset. The above process is repeated until all tomatoes in the operation area are picked, after which the 
robot continues to move along the path until the termination. The workflow of the robot is shown in Figure 2.

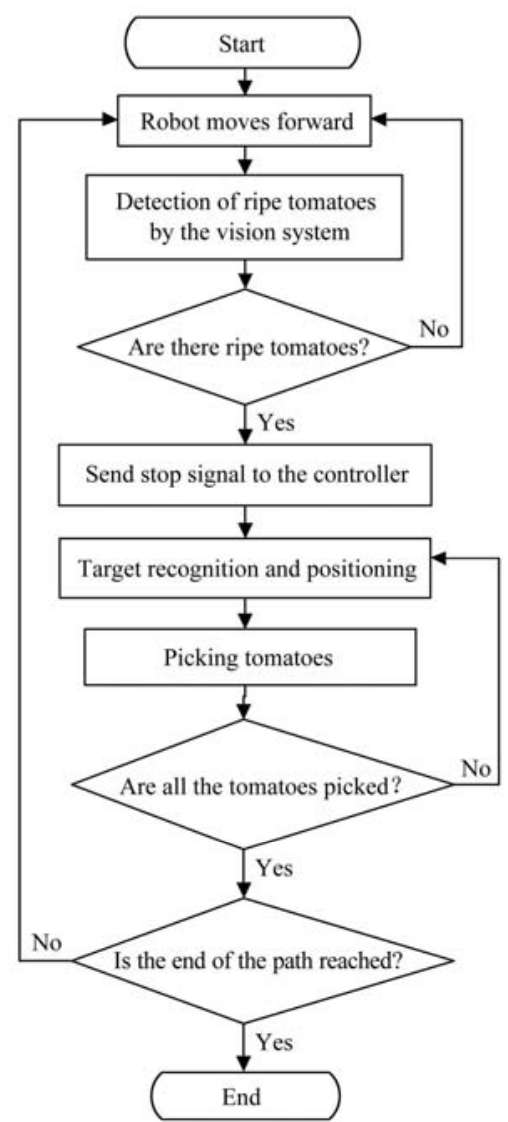

Figure 2 Workflow of the tomato harvesting robot

\section{General design of the robot}

\subsection{Four-wheel independent steering chassis}

A four-wheel independent steering chassis with a high ground clearance was used in this study. This chassis was driven by several DC servo motors and consisted mainly of chassis frame, a power supply system, and an electronic control system. The load bearing capacity of the chassis is higher than $100 \mathrm{~kg}$, the dead weight of the robot is about $540 \mathrm{~kg}$, and the maximum velocity during operation is about $3.6 \mathrm{~km} / \mathrm{h}$. Embedded systems were used for the controlling of the chassis, which communicated with the main control system via the controller area network (CAN) bus ${ }^{[12,13]}$. Two operating modes were available. One was the remote control mode whereby the robot was controlled manually for the transport of the robot to another place, and the other one was the automatic mode whereby the robot was controlled by the main control system for automatic picking of tomatoes in complex working environment.

\subsection{Five-DOF manipulator}

The 5-DOFmanipulator was mainly composed of a mechanical arm and an end effector. Because this robot was designed mainly for the harvesting of tomatoes in the greenhouse with a very limited workspace, a dexterous 4-DOF mechanical arm and a 1-DOF end effector are arranged in tandem. The mechanical arm was composed mainly of the base, shoulder joint, elbow joint and wrist joint.

A major drawback of most end effectors is their poor portability and flexibility ${ }^{[14]}$. In this study, a shear type gripper was designed to pick tomatoes, which gave equal consideration to the stability of the gripper and the complexity of the structural control. The pitching actions involve the grasping, cutting and detaching of tomatoes. The gripper was composed mainly of telescopic cylinder, air pump, magnetic valve, relay and shear. As the tomato is very soft and fragile, wrong direction and position are likely to cause rupture or bruising to the tomato. Thus, the image recognition of the gripper needs to be precisely controlled.

\subsection{Control system}

\subsubsection{Laser navigation control system}

\subsubsection{Hardware}

The hardware of the control system was designed based on modular robotic system (MRS), which was composed of a number of modules such as central processor, LMS151 laser scanner, end effector, host computer and remote controller. The end effector module is the key component of this control system, which can be divided into the core control module (CCM) and the extended interface module (EIM). A PIC18F25K80 microcontroller (Microchip Technology Inc., USA) was used in the CCM, and the power converter module and dual-channel digital isolators ADUM1200/ADUM1201 were used to provide CAN bus communication, pulse width modulation (PWM) output, and the isolation between microcontroller and the motor control system. Compared with conventional photoelectric coupling isolation, dual-channel digital isolators were characterized by higher data transmission rate, high transient common mode rejection, and DC correction. 


\subsubsection{Navigation control strategies}

LMS151 laser scanner (SICK, Germany) was used in this study, which operated at a resolution of $0.5^{\circ}$ for a coverage of $270^{\circ}$ with 541 readings in each scan. It is a kind of distance measurement. The maximum scanning distance is $50 \mathrm{~m}$, and the optional angular resolution is $0.25^{\circ}$ or $0.50^{\circ}$. The laser receiver can receive the reflected laser wave, and thus the laser radar can calculate the distance between the object and the scanner. This makes it easy to determine the silhouette and coordinate position of the target, which provide necessary information for automatic navigation. The major purpose of feature extraction is to transform the original data from a higher dimension to a lower dimension with most of the desired information content preserved. The information of the angles and the corresponding distances can be extracted from the original data collected by the sensors. The measured data of the sensor is in the polar form; and the returned data is the relative angle with scanner and the distance between the scanner and object. A finite set of data pairs $\left(x_{\mathrm{i}}, y_{\mathrm{i}}\right)$ can be obtained by using coordinate transformation, and then fitted to a straight line using the least square method. The four-wheel independent steering system enables the robot to move automatically along the predetermined path. The mechanical part is designed based on the Ackerman steering model, which allows the four wheels to turn about a common center at differential velocities ${ }^{[15]}$. For a low-speed steering, the two front wheels of the robot turn in a direction opposite to that of the two rear wheels, and the robot has a small turning radius, as shown in Figure 3.

In Figure 3, $O$ is the barycentre in the ideal state; $O^{\prime}$ is the instant center; $\beta_{i}(i=A, B, C, D)$ is the deflection angle of the wheel $\left({ }^{\circ}\right) ; R_{i}(i=A, B, C, D)$ is the turning radius of the wheel about the instant center $(\mathrm{m}) ; V_{i}(i=A, B, C, D)$ is the linear velocity of the wheel about the instant center $(\mathrm{m} / \mathrm{s})$; $R$ is the turning radius of the wheel about the barycentre $(\mathrm{m}) ; \omega$ is the angular velocity of the robot ( $\mathrm{rad} / \mathrm{s}) ; r$ is the distance between the left wheel and the instant center (m); $a$ and $b$ are the horizontal distance between the extended line of the instant center and the front and rear axle (m); $W$ is the distance between the left and right wheels (m); and $L$ is the wheelbase (m). A single chip microcomputer and the proportional-integral-derivative (PID) algorithm are used in the control system for the four-wheel independent steering system. The steering angles of the front and rear wheels can be precisely controlled by adjusting the PWM output of their respective driving motors. The three parameters of PID controller are tuned, where the proportional coefficient $P=3$, the integration time constant $T_{i}=1.25 \mathrm{~ms}$, and the differential time constant $T_{d}=0.3 \mathrm{~ms}$, under which the wheels can reach a steady state after 2-3 oscillations, and the steering angle can be precisely controlled.

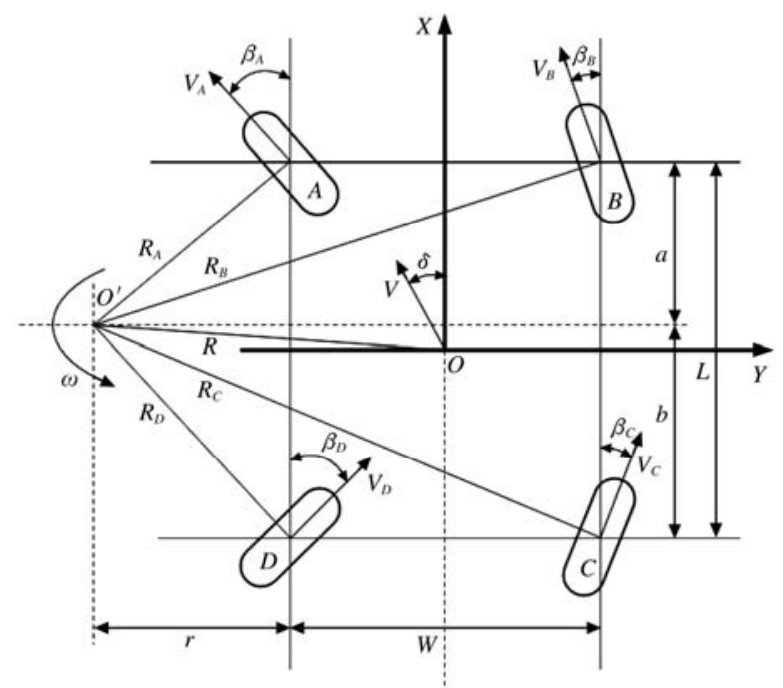

Figure 3 Low-speed steering based on Ackerman steering model

The measurement data recorded by the laser sensor are fitted to a straight line using the least square method. Figure 4 shows the installation location of the laser radar. The obstacle contours at both sides of the path are obtained, and the line through the center of these contours is determined as the navigation $A B$ line, as shown in Figure 5, where the abscissa is the spacing between two adjacent rows of tomatoes $(\mathrm{mm})$, and the ordinate is the moving distance $(\mathrm{cm})$.

If the tomato harvesting robot deviates from the predetermined navigation path, the controller will compute the lateral deviation and the course deviation. The adaptive PID algorithm is used to adjust the parameters to guide the movement of the robot along the navigation path. The mobile platform angle control process is as follows: the current angle value minus the set point is the $\Delta \theta$. If $\theta \geq 15^{\circ}$, adjust the rotation of wheel clockwise quickly; if $\theta<-15^{\circ}$, adjust the rotation of wheel 
counter clockwise; if $-15^{\circ}<\theta<15^{\circ}$, adjust the rotation of wheel slowly; if $\theta>0.065^{\circ}$, adjust the rotation of wheel clockwise slowly; if $\theta<-0.065^{\circ}$, adjust the rotation of wheel counter clockwise slowly; if $-0.065^{\circ}<\theta<0.065^{\circ}$, wheels stop running. This ensures the precision and stability of the wheels, and wheel jitter can be prevented.

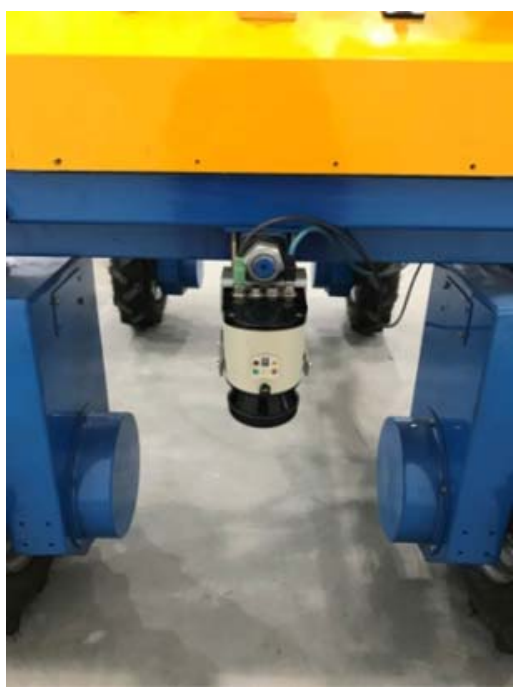

Figure 4 Installation location of the laser scanner

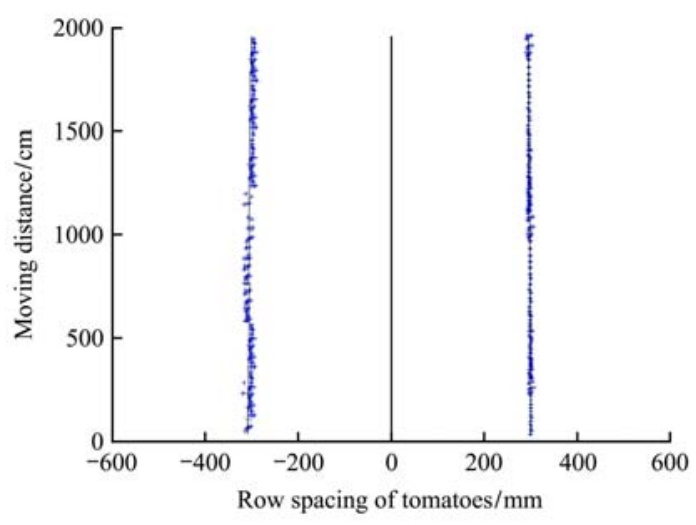

Figure 5 Navigation $\mathrm{AB}$ line

\subsubsection{Binocular stereovision system}

\subsubsection{Algorithm for image preprocessing}

The point greyGS3 U3-15S5C camera (Canada) is used to acquire tomato image. The distance between the target and camera is $40-100 \mathrm{~cm}$. The image resolution is $1384 \times 1032$. The recognition process of ripe tomatoes is as follows: the binocular camera is calibrated by the method proposed by Zhengyou Zhang, and the internal parameters and the relative position of camera are calculated, and then the threshold segmentation is used to segment the preprocessed gray scale image. Eventually the target is identified. This can lay a foundation for subsequent three-dimensional positioning and picking of tomatoes. Gray-level images are obtained using the normalized color difference method, and segmented into foreground and background using the Otsu algorithm. The light intensity information in the red and green components can be eliminated by the normalized color difference method ${ }^{[16,17]}$ :

$$
N R G(x, y)=\frac{R(x, y)-G(x, y)}{R(x, y)+G(x, y)}
$$

where, $R(x, y)$ and $G(x, y)$ are the red and green components of the pixel $(x, y)$ in the image. The gray values obtained by the above formula range from -1 to 1 , which is normalized as:

$$
N R G^{\prime}(x, y)=(N R G(x, y)+1) / 2 \times 255
$$

Figure 6 shows that the Otsu algorithm can effectively extract the target of interest in the image, which contributes significantly to subsequent target recognition and the reduction in computation time ${ }^{[18,19]}$. However, this method may fail to segment overlapping tomatoes into individual ones. This problem can be solved with the use of an elliptic template. More specifically, the gray-level picture is obtained by the weighted average method, and the putative target is matched with the elliptic template. When the center of the elliptic template is located in the region obtained by the Otsu algorithm, this ellipse is determined to be a tomato ${ }^{[20]}$, otherwise it is not a tomato.

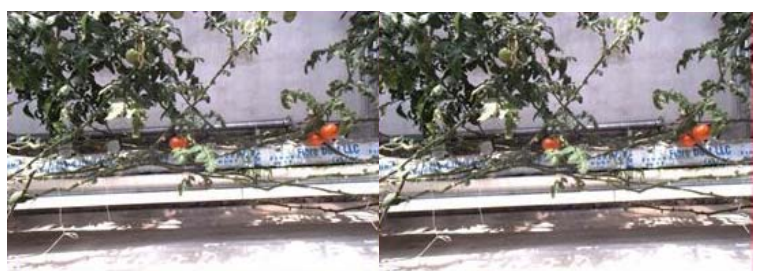

a. Original image (left camera and right camera)

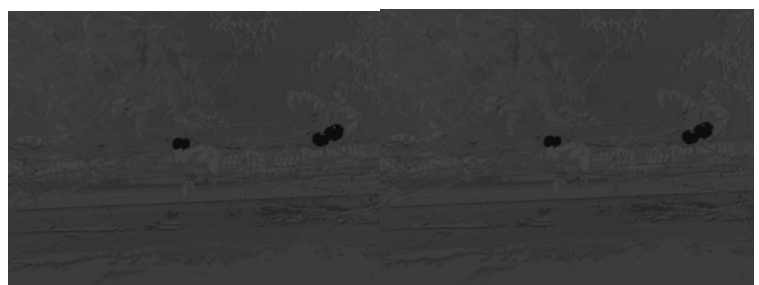

b. Normalized difference of red and green graying (left camera and right camera)

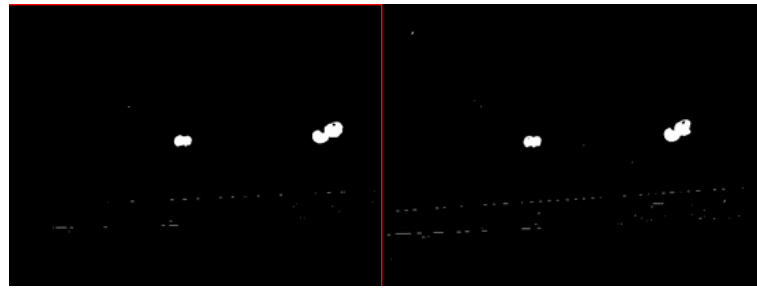

c. Otsu segmentation results (left camera and right camera)

Figure 6 Tomato image processing 


\subsubsection{Positioning of tomatoes}

A rectangular region of the color image that contains all the tomatoes is selected, and then the Harris points extracted from the left and right images are matched. The three-dimensional coordinates of the matched points are obtained from the following Equations. The distances between all matched points to the binocular camera are measured, and the average is used to represent the actual distance of the tomato.

$$
\begin{aligned}
& X=-Z\left(\text { width } / 2-x_{l}\right) / f \\
& Y=-Z\left(\text { height } / 2-y_{l r}\right) / f \\
& Z=f_{b} /\left(x_{l}-x_{r}\right) \\
& \text { Dist }=\operatorname{sqrt}\left(x^{2}+y^{2}+z^{2}\right)
\end{aligned}
$$

where, $f$ is the focal length and $b$ is the baseline distance, both of which can be obtained from the $\mathbf{Q}$ matrix; $x_{l}$ and $x_{r}$ are the $x$-axis coordinates of the matched point in the left and right pictures; $y_{l r}$ is the average of the $y$-axis coordinates of the matched points; height and width are the height and width of the picture, respectively. When $f=767, b=76 \mathrm{~mm}$, the elliptic template radius is 25 pixels, and ripe tomatoes can be recognized. The matching results are shown in Figure 7.

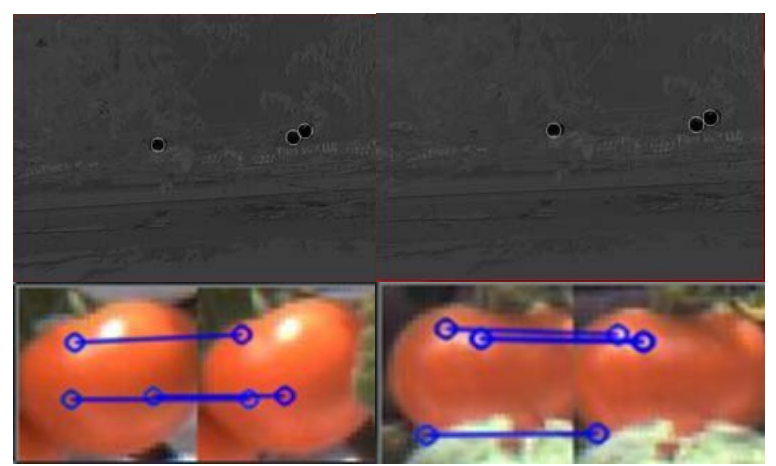

Figure 7 Matching results of regional characteristics

\subsubsection{Control strategies of the manipulator}

The harvesting robot will inevitably come across a variety of obstacles, mainly including stems, immature tomatoes, and supporting robs. In this study, obstacle avoidance strategies are designed based on the $\mathrm{C}$-space $\operatorname{method}^{[21]}$. The horizontal and vertical planes $(\mathrm{H}-$ and V-plane) are used to characterize 3D C-space obstacles based on the re-projection method, where the H-plane is perpendicular to the rotation axis of the manipulator waist, while the V-plane is perpendicular to the H-plane and intersects or parallels the rotation axis of the waist. The results show that all joints are in the same plane except
Joint 1, and the mechanical arm is equivalent to a cylinder and projected as a rectangle in the H-plane. The circles in the H-plane represent the immature tomatoes, and the critical collision angle is shown in Figure 8a. The rectangles in the H-plane represent those rectangular obstacles such as the stems or supporting robs, and the critical collision angle is shown in Figure $8 \mathrm{~b}$. The other joints are shown in Figure 8c.

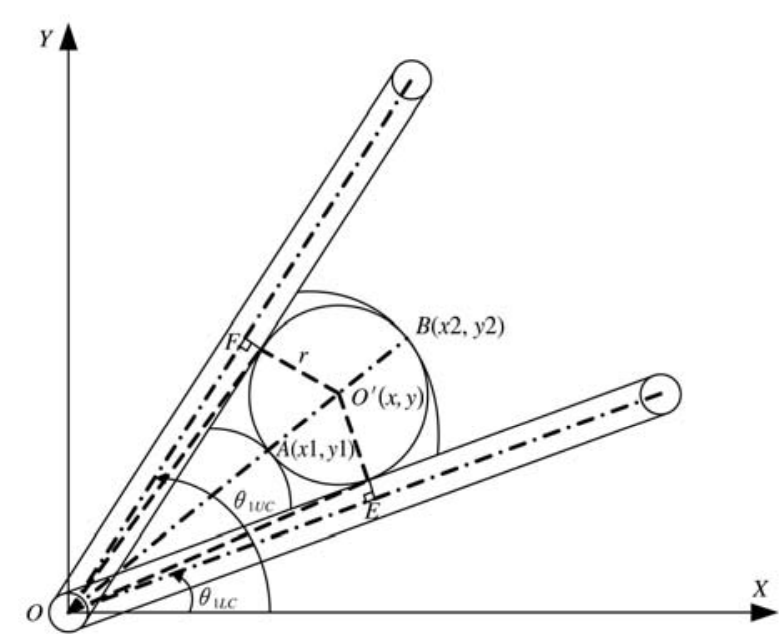

a. Critical collision angle of Joint 1 for circular obstacles

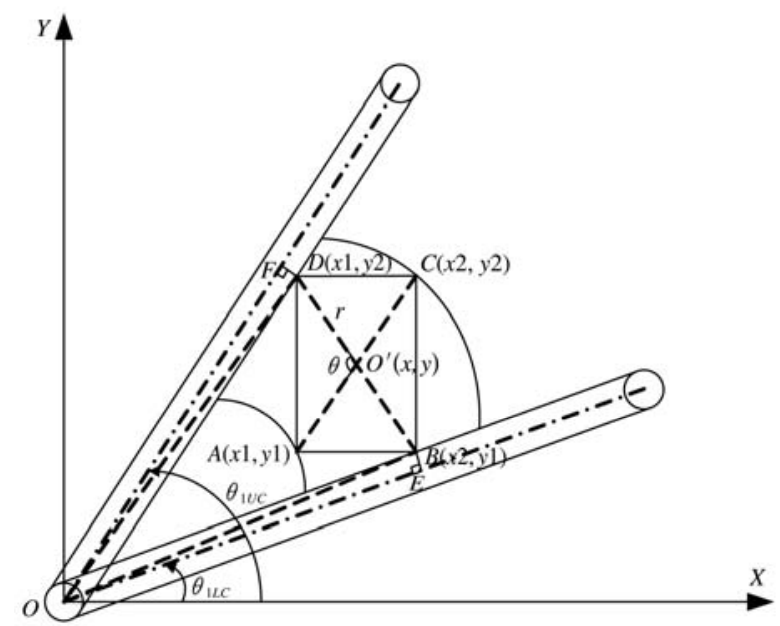

b. Critical collision angle of Joint 1 for rectangular obstacles

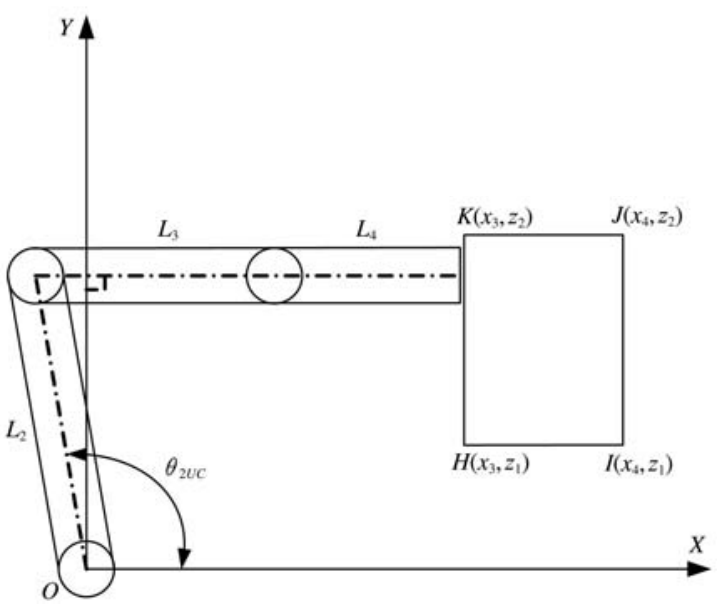

c. Critical collision angles of other joints

Figure 8 Critical collision angles of each joint 
The motion of Joint 1 and Joint 2 is planned in the two-dimensional C-space based on the critical collision angles, and that of Joint 3 is planned from the starting point to the target point according to the motion points of Joint 1. This approach can effectively improve the search efficacy and thus be more suitable for practical applications.

The grid method is used to describe the geometry of the $\mathrm{C}$-space and the $\mathrm{A}^{*}$ algorithm is used for path finding. As the C-space is discretized and the search step is greater than 1 degree, the resultant path is usually a broken line. This is not allowed for the manipulator because of the interruption of the movement at the turning point of the broken line. However, as the broken line obtained by the $A^{*}$ algorithm is the optimal with some points near the critical collision point, collision is more likely to occur between the fitted points obtained by simple curve fitting and the obstacles. This problem can be solved by curve smoothing, and the results are shown in Figures 9a and 9b.

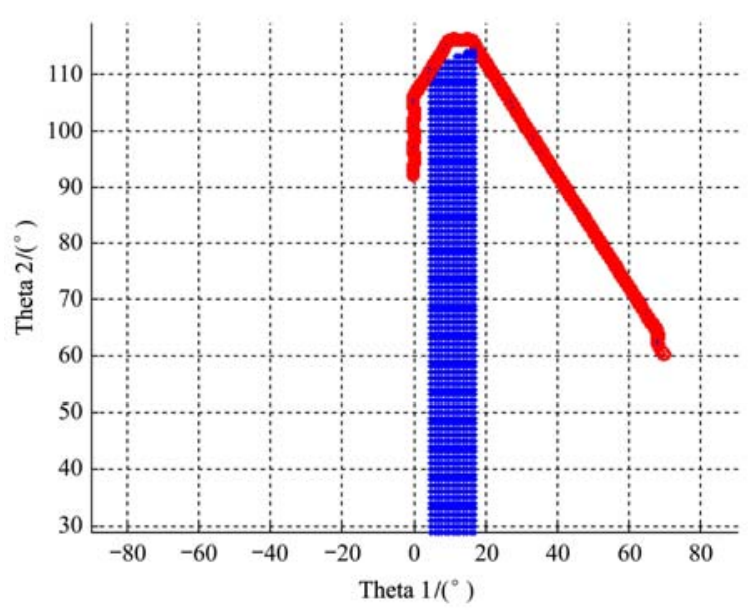

a. 2D C-space path planning of Joint 1 and 2

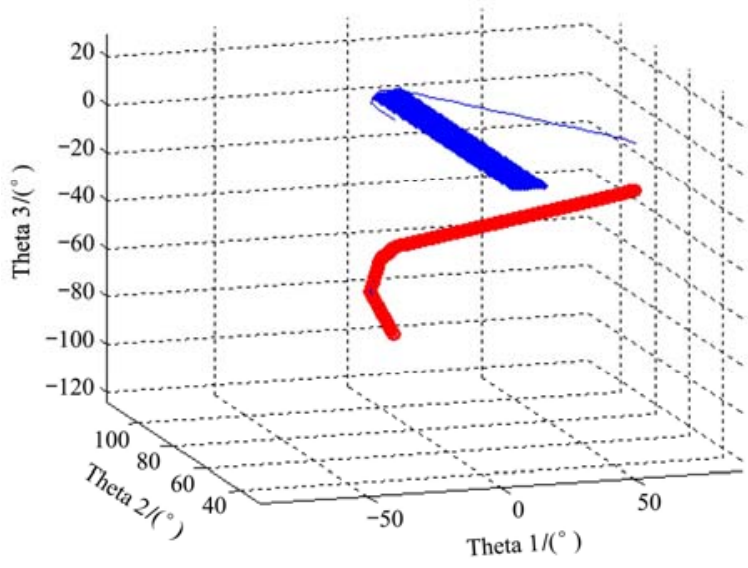

b. Path planning of joint 3

Figure 9 Path planning of each joint

\section{Experimental results and analysis}

\subsection{Control of steering angle}

The performance of the four-wheel independent steering system in controlling the steering angles was evaluated. The robot was allowed to move on a level road, and different steering angles $\left(0^{\circ}-360^{\circ}\right)$ were set through the host computer. Each angle was tested for 10 times. Table 1 shows that the maximum average absolute error between the set angle and the actual angle is about $0.14^{\circ}$,and the maximum standard deviation is about $0.04^{\circ}$, indicating that the steering angles of the wheels can be precisely and reliably controlled.

Table 1 Results of the angle control tests

\begin{tabular}{cccc}
\hline $\begin{array}{c}\text { Angle setting } \\
/\left(^{\circ}\right)\end{array}$ & $\begin{array}{c}\text { Average angle } \\
/\left(^{\circ}\right)\end{array}$ & $\begin{array}{c}\text { Average absolute } \\
\text { error } /\left(^{\circ}\right)\end{array}$ & $\begin{array}{c}\text { Standard deviation } \\
\sigma /\left(^{\circ}\right)\end{array}$ \\
\hline 0 & 0.05 & 0.05 & 0.04 \\
10.20 & 10.29 & 0.09 & 0.02 \\
20.50 & 20.54 & 0.04 & 0.01 \\
84.80 & 84.74 & 0.06 & 0.02 \\
90.00 & 90.14 & 0.14 & 0.03 \\
280.60 & 280.63 & 0.03 & 0.01 \\
356.00 & 356.07 & 0.06 & 0.01 \\
\hline
\end{tabular}

\subsection{Navigation tracking tests}

The robot moved for about 5 mat a velocity of less than $0.5 \mathrm{~m} / \mathrm{s}$, and the vertical deviation between the navigation line and the actual path was measured at 100 sampling points using a meter rule. Figure 10 shows that there is a large deviation of about $1.54 \mathrm{~m}$ between the starting point of the robot and the predetermined path. However, the laser navigation system is able to rapidly and accurately track the path, with the deviation being less than $8 \mathrm{~cm}$, thus indicating that this system has good control precision and stability.

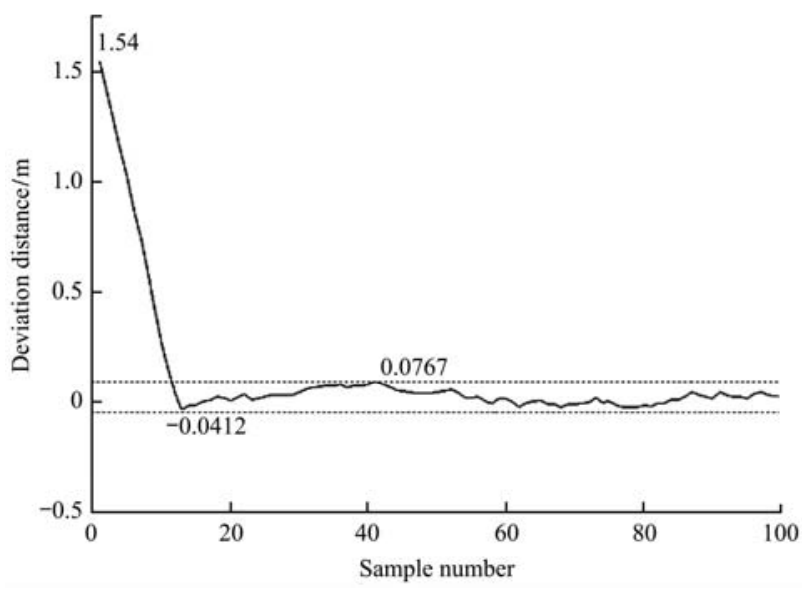

Figure 10 Deviation of the harvesting robot 
The experimental data were then analyzed to verify the dynamic response of the navigation control system. The motor speed was set to $100 \mathrm{r} / \mathrm{s}$, and the motor speed was sampled. The dynamic response curve can be obtained in the process of speed control, as shown in Figure 11.

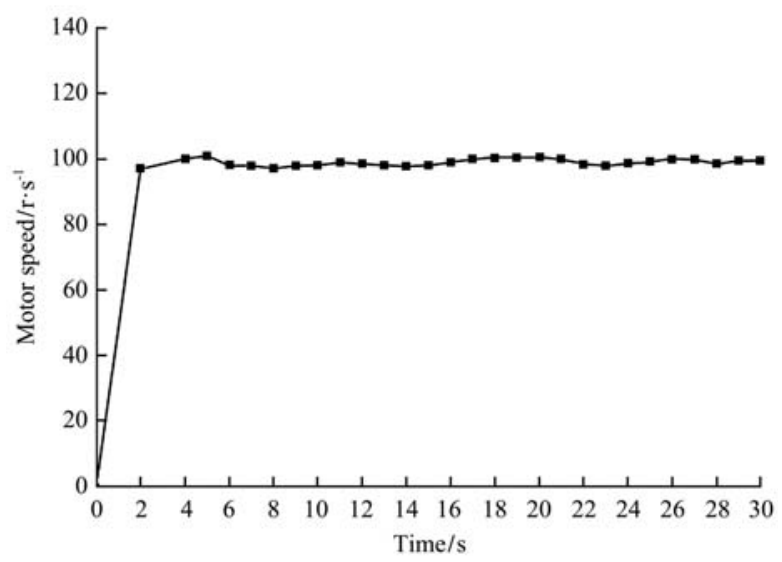

Figure 11 Dynamic response curve

The dynamic response process, which is also known as the transition process, can be seen from the diagram. It refers to the change from the initial state to set condition. The dynamic performance of the control system was evaluated using the following parameters:

(1) The delay time $t_{\mathrm{d}}$ refers to the time for the response curve to reach $50 \%$ of its steady-state value. The $t_{\mathrm{d}}$ obtained by calculating the motor speed in the interval of $[0,50]$ is $1.23 \mathrm{~s}$.

(2) The rising time $t_{\mathrm{r}}$ refers to the time for the steady-state value to raise from $10 \%$ to $90 \%$. The $t_{\mathrm{r}}$ obtained by calculating the motor speed value in the interval of $[10,90]$ is $1.48 \mathrm{~s}$.

(3) The peak time $t_{\mathrm{p}}$ refers to the time to reach the first peak which exceeds its steady-state value. The $t_{\mathrm{p}}$ obtained by calculating the motor speed value in the interval of $[0,100.5]$ is $4.89 \mathrm{~s}$.

(4) Overshoot amount $\sigma \%$ :

$\sigma \%=\frac{h(t p)-h(\infty)}{h(\infty)} * 100 \%=\frac{100.5-100}{100}=0.005=0.5 \%$

(5) Adjust time $t_{\mathrm{s}}$ refers to the minimum time which no longer goes beyond error. The $t_{\mathrm{s}}$ obtained by calculating the motor speed value in the interval of $[0,99.8]$ is $5.97 \mathrm{~s}$.

The experimental results clearly show that as the speed set is about 2-3 s, the motor can enter a state of stability control and reach the set rotation speed. It shows that the control system has good walking speed control effect.

\subsection{Load-bearing capacity of the end effector}

To evaluate the load-bearing capacity of the mechanical arm, several 500-g weights $(0.5 \mathrm{~kg}, 1 \mathrm{~kg}$ and $1.5 \mathrm{~kg}$ ) were placed on the end effector, and the manipulator moved in the $x$-axis direction by $-300 \mathrm{~mm}$ to $300 \mathrm{~mm}$, in the $y$-axis direction by $-400 \mathrm{~mm}$ to $400 \mathrm{~mm}$, and in the $z$-axis direction by $-300 \mathrm{~mm}$ to $300 \mathrm{~mm}$, and the stability of the manipulator movement of the was observed. The experimental environment is shown in Figure 12, and the results under the three load conditions are shown in Figures 11a-11c, respectively.

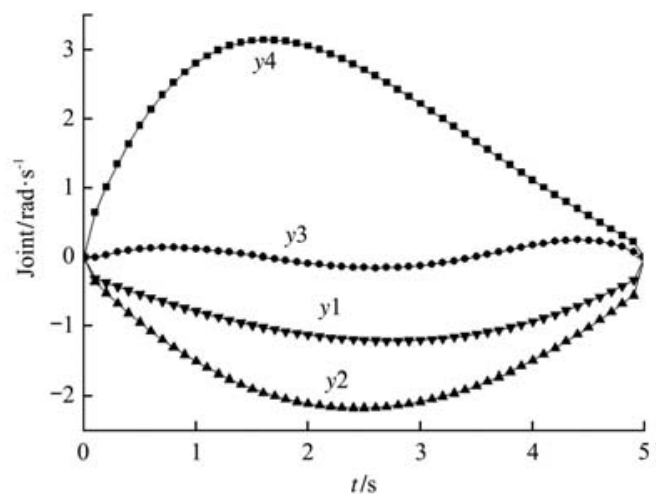

a. Angular velocity curve of each joint under a load of $0.5 \mathrm{~kg}$

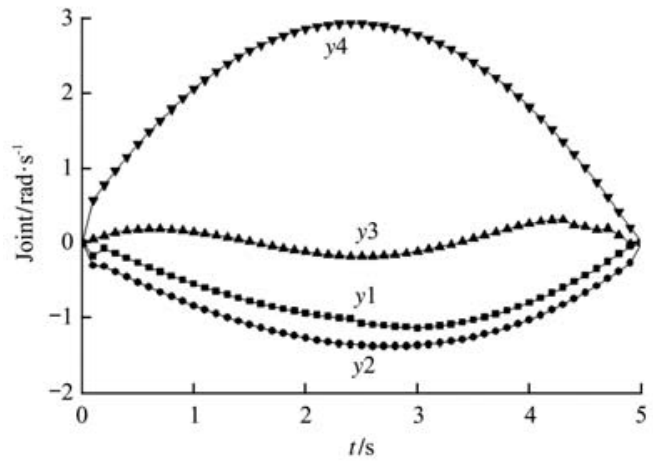

b. Angular velocity curve of each joint under a load of $1 \mathrm{~kg}$

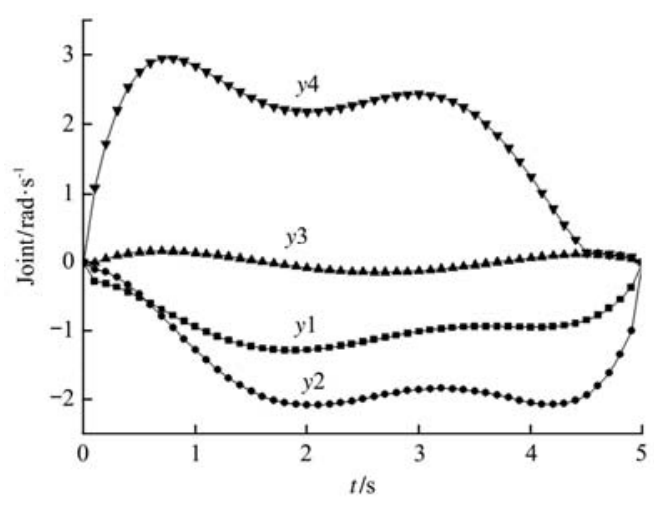

c. Angular velocity curve of each joint under a load of $1.5 \mathrm{~kg}$

Figure 12 Angular velocity curve of each joint under different loads 
The results clearly show that under the load of $0.5 \mathrm{~kg}$ and $1 \mathrm{~kg}$, all the joints have very good tracking precision, with a smooth change in the angular velocity and no abnormality in the movement of each joint. However, under the load of $1.5 \mathrm{~kg}$, there is a large variation in the angular velocity of Joint 2 and Joint 4, but the load bearing capacity of the manipulator can meet the need required for the picking of tomatoes.

\subsection{Recognition rate of ripe tomatoes}

Under different light intensities (different times of the same day) and backgrounds (random), 300 static images $(1384 \times 1032$ pixels $)$ of the tomato field are captured to evaluate the success rate of the binocular vision system in recognizing ripe tomatoes. Of the 300 pictures, a total of 298 pictures have been successfully recognized, with an average recognition time of $1.5 \mathrm{~s}$ per picture, and the success rate is shown in Table 2. The failure can be attributed to that: 1) ripe tomatoes may overlap with each other; and 2) the tomato of interest may be sheltered by obstacles such as immature fruits, stems and leaves.

Table 2 Success rate of the binocular stereovision system in the recognition of ripe tomatoes

\begin{tabular}{cccc}
\hline Time & Success & Failure & Success rate $/ \%$ \\
\hline $10: 00$ & 299 & 1 & 99.8 \\
$12: 00$ & 298 & 2 & 99.3 \\
$15: 00$ & 300 & 0 & 100 \\
\hline
\end{tabular}

\subsection{Positioning error of ripe tomatoes}

The center of the left camera was taken as the origin of the geodetic coordinate system. Dynamic tests were performed at different positions for 100 times, and the difference between the theoretical values and the actual results given by the vision system was taken as an indicator of the absolute error, as shown in Figure 13. It shows that when the distance is less than $600 \mathrm{~mm}$, the positioning error is less than $10 \mathrm{~mm}$ except some singular points.

\subsection{Success picking rate}

The picking tests were performed for 100 times in the greenhouse (Figure 14), and the success rate is shown in Table 3. The failure can be attributed to: 1) light intensity; 2) the overlapping or sheltering of obstacles such as immature fruits, stems and leaves; and 3) the difference between the motion trajectory determined by the algorithm and the actual target positions.

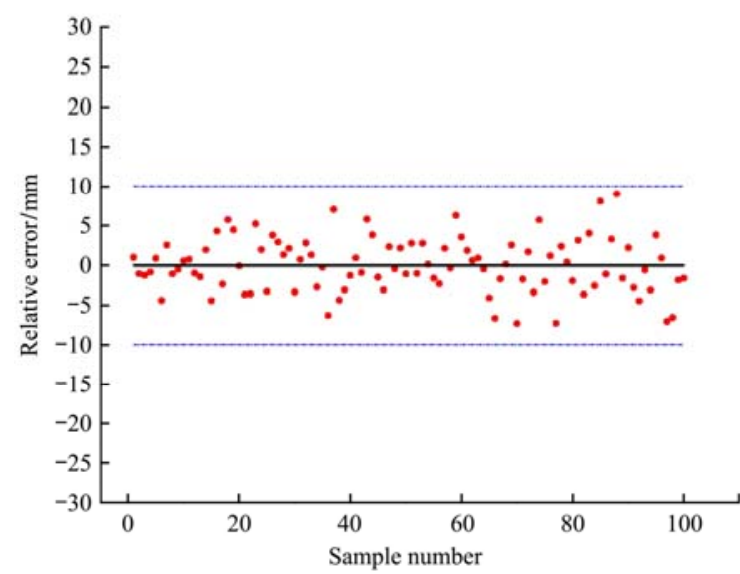

Figure 13 Orientation error analysis of ripe tomatoes

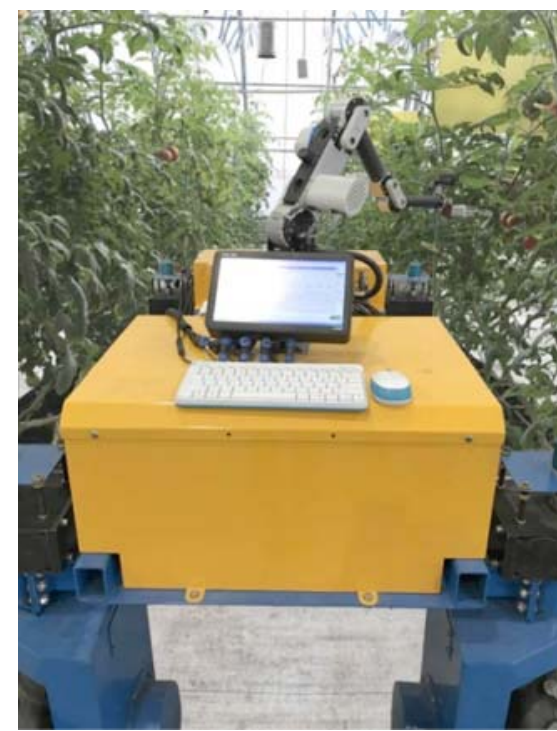

Figure 14 Experimental environment of tomato harvesting robot

Table 3 Success rate of the pitching tests

\begin{tabular}{cccc}
\hline No. & Success & Failure & Success rate/\% \\
\hline $1-50$ & 43 & 7 & 86 \\
$51-100$ & 44 & 6 & 88 \\
\hline
\end{tabular}

\section{Conclusions}

A robot was designed for harvesting ripe tomatoes in the greenhouse in this study, which consisted mainly of a four-wheel independent steering chassis, a 5-DOF harvesting system, a navigation system, and a binocular vision system.

The low-speed steering of the four-wheel independent steering system was achieved based on Ackerman steering model, and the PID algorithm was used in the control system. This robot has high fault tolerance and environmental adaptability. The maximum average absolute error between the set angle and the actual angle is about $0.14^{\circ}$, and the maximum standard deviation is about $0.04^{\circ}$. The laser navigation system is able to 
rapidly and accurately track the path, with the deviation being less than $8 \mathrm{~cm}$.

The Otsu algorithm and the elliptic template method are used in the automatic recognition of ripe tomatoes, and obstacle avoidance strategies are designed based on the C-space. The path obtained by the $\mathrm{A}^{*}$ algorithm is further smoothed, resulting in safe and highly efficient obstacle avoidance. The load bearing capacity of the mechanical arm is $1.5 \mathrm{~kg}$. The success rate of the binocular vision system in the recognition of ripe tomatoes is $99.3 \%$. When the distance is less than $600 \mathrm{~mm}$, the positioning error is less than $10 \mathrm{~mm}$. The time needed from recognition to pitching is about $15 \mathrm{~s}$ per tomato, with a success rate of about $86 \%$.

\section{Acknowledgements}

This study was financially supported by the National 863 planning project of China- digital design and intelligent control technology of agricultural facilities equipment (2013AA102406) and the Beijing municipal science and technology project (Z161100004916118).

\section{[References]}

[1] National Bureau of Statistics of China. 2012 China Statistical Yearbook. Beijing: China Statistics Press, 2012. (in Chinese)

[2] Xiang R, Ying Y B, Jiang H Y. Development of real-time recognition and localization methods for fruits and vegetables in field. Transactions of the CSAM, 2013; 44(11): 208-219. (in Chinese)

[3] Li H, Xu L. The development and prospect of agricultural robots in China. Acta Agriculturae Zhejiangensis 2015; 27(5): 865-871.

[4] Kondo N, Yata K, Iida M, Shiigi T, Monta M, Kurita M, Omori H. Development of an end-effector for a tomato cluster harvesting robot. Engineering in Agriculture. Environment and Food, 2010; 3(1): 20-24.

[5] Feng Q C, Cheng W, Zhou J J, Wang X. Design of structured-light vision system for tomato harvesting robot. Int J Agri \& Biol Eng, 2014; 7(2): 19-26.

[6] Rath T, Kawollek M. Robotic harvesting of Gerbera Jamesonii based on detection and three-dimensional modeling of cut flower pedicels. Computers and Electronics in Agriculture, 2009; 66: 85-92.

[7] Niu W, Lang L, Jing Q, Wang D. Research on robot manipulator uncalibrated visual serving under two-camera bi-axial parallel vision configuration. Advances in Mechanical Engineering, 2015; 7(4): 1-14.
[8] Xiang R, Jiang H Y, Ying Y B. Recognition of clustered tomatoes based on binocular stereo vision. Computers and Electronics in Agriculture, 2014; 106: 75-90.

[9] Ding H, Li H X. Fuzzy avoidance control strategy for redundant manipulators. Engineering Applications of Artificial Intelligence, 1999; 12: 513-521.

[10] Pi Y J, Wang X Y. Trajectory tracking control of a 6-DOF hydraulic parallel robot manipulator with uncertain load disturbances. Control Engineering Practice, 2011; 19(2): 185-193.

[11] Joel Perez P, Perez J P, Soto R, Flores A, Rodriguez F, Meza J L. Trajectory tracking error using PID control law for two-link robot manipulator via adaptive neural networks. The 2012 Iberoamerican Conference on Electronics Engineering and Computer Science: Procedia Technology, 2012; 3: 139-146.

[12] Zhao J, Cui X D, Zhu Y H, Tang S F. A new reconfigurable modular robotic system with multimode locomotion ability. International Journal, 2012; 4(2): 178-190.

[13] Tangerino G T, Godoy E P, Tabile R A, Inamasu R Y, Porto A J V. Hydraulic networked control of four wheel steering agricultural robot. International Conference on Control and Automation. America: IEEE, 2011; pp.142-147.

[14] Liu J Z, Li P P, Li Z G. Hardware design of the end-effector for tomato-harvesting robot. Transactions of the CSAM, 2008; 39(3): 109-112. (in Chinese)

[15] Ko M H, Ryuh B S, Kim K C, Suprem A, Mahalik N P. Autonomous greenhouse mobile robot driving strategies from system integration perspective: review and application. Transactions on Mechatronics. America: IEEE/ASME, 2014; pp.1-12.

[16] Pearson T. Hardware-based image processing for high-speed inspection of grains. Computers and Electronics in Agriculture, 2009; 69(1):12-18.

[17] Okamoto H, Lee W S. Green citrus detection using hyperspectral imaging. Computers and Electronics in Agriculture, 2009; 66(2): 201-208.

[18] Si Y S, Li G, Feng J. Location of apples in trees using stereoscopic vision. Computers and Electronics in Agriculture, 2015; 112: 58-74.

[19] Song S E, Cho N, Tokuda J, Hata N, Tempany C, Fichtinger $\mathrm{G}$, et al. Preliminary evaluation of a MRI-compatible modular robotic system for MRI-guided prostate interventions. International Conference on Biomedical Robotics and Biomechatronics. America: IEEE RAS \& EMBS, 2010; pp.796-801.

[20] Boryga M, Grabos A, Kolodziej P. Trajectory planning with obstacles on the example of tomato harvest. Agriculture and Agricultural Science Procedia, 2015; 7: 27-34.

[21] Min H S, Lin Y H, Wang S J, Wu F, Shen X. Path planning of mobile robot by mixing experience with modified artificial potential field method. Advances in Mechanical Engineering, 2015; 7(12): 1-17. 